\title{
Inch-Scale Ball-in-Bowl Plasmonic Nanostructure Arrays for Polarization-Independent Second Harmonic Generation
}

Xian-Xin Wu, ${ }^{1,2}$ Wen-Yu Jiang,, ${ }^{2,3}$ Xiao-Feng Wang,, ${ }^{2,3}$ Li-Yun Zhao,, ${ }^{4,5}$ Jia Shi, ${ }^{1,2}$ Shuai Zhang, ${ }^{1,2}$ Xinyu Sui, ${ }^{1,2}$ Zhe-Xue Chen, ${ }^{2,3}$ Wen-Na Du, ${ }^{1,6}$ Jian-Wei Shi, ${ }^{1}$ Qian Liu, ${ }^{2,3}$ Qing Zhang, ${ }^{4,5}$ Yong Zhang, ${ }^{2,3, *}$ and Xin-Feng Liu ${ }^{1,2, *}$

${ }^{1}$ CAS Key Laboratory of Standardization and Measurement for Nanotechnology, CAS Center for Excellence in Nanoscience, National Center for Nanoscience and Technology, Beijing 100190, P. R. China

${ }^{2}$ University of Chinese Academy of Sciences, Beijing 100049, P. R. China

${ }^{3}$ CAS Key Laboratory of Nanosystem and Hierarchical Fabrication, CAS Center for Excellence in Nanoscience, National Center for Nanoscience and Technology, Beijing 100190, P. R. China

${ }^{4}$ Department of Materials Science and Engineering, College of Engineering, Peking University, Beijing 100871, China

${ }^{5}$ Research Center for Wide Band Semiconductor, Peking University, Beijing 100871, China

${ }^{6}$ Key Laboratory of Semiconductor Materials Science, Beijing Key Laboratory of Low Dimensional Semiconductor Materials and Devices, Institute of Semiconductors, Chinese Academy of Sciences, Beijing, 100083, China

\section{Corresponding Author}

*Email address: liuxf@nanoctr.cn, zhangyong@nanoctr.cn 


\section{Table of Contents}

Figure S1: Plasmonic nanostructure array with different size.

Figure S2: Schematic diagram of the SHG measurement.

Figure S3: Power dependence of SHG intensity.

Figure S4: Nonlinear generation from the plasmonic array under applied strain.

Figure S5: Cross-section TEM pictures of the hybrid structure sample.

Figure S6: Wavelength-dependent SHG of Hybrid structures.

Figure S7: Polarization-dependent characterization of the hybrid nanostructure array.

Figure S8: Comparisons in simulated electric field intensity distribution among different hybrid nanostructure

Figure S9: Real-space images of SHG from hybrid nanostructure array at different tilt-angle.

Figure S10: Tilt-dependent SHG signals of different samples.

Figure S11: Optical setup for angle-resolved spectroscopy by spatially-resolved Fourier imaging.

Figure S12: Effective second-order nonlinear susceptibility $\chi^{(2)}$ of the structure array.

Note1: Nonlinear response of the hybrid nanostructure array.

Note2: Calculation of effective second-order nonlinear susceptibility 
a

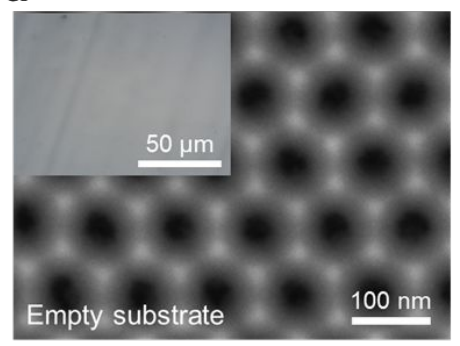

d

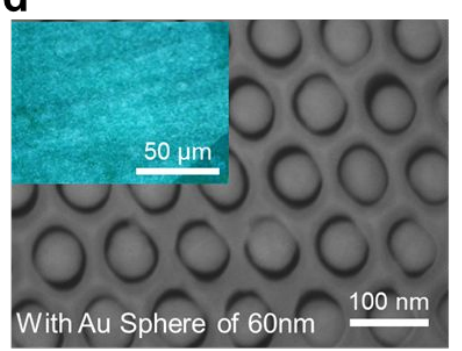

g

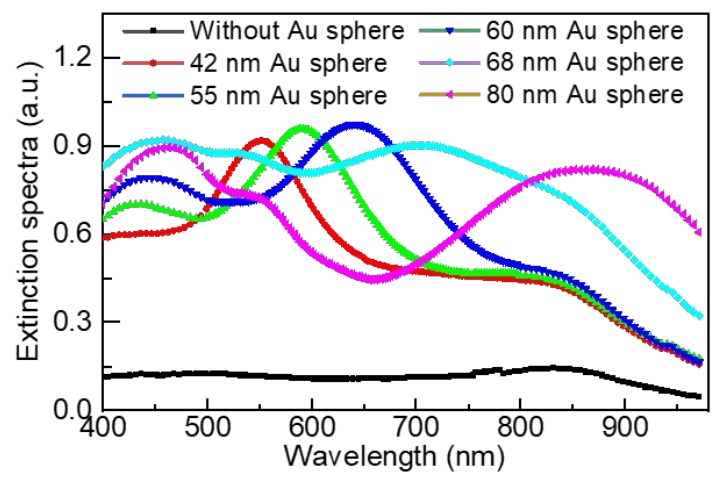

b

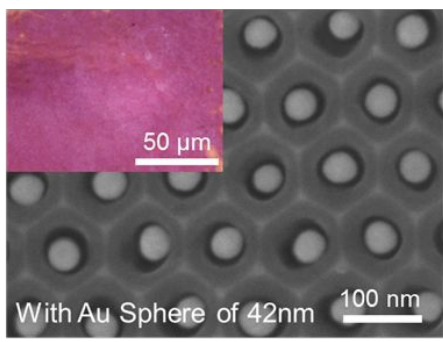

e

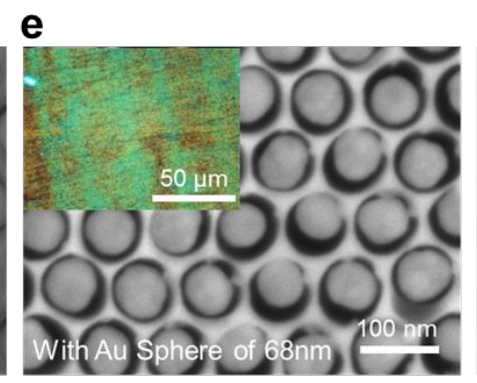

C
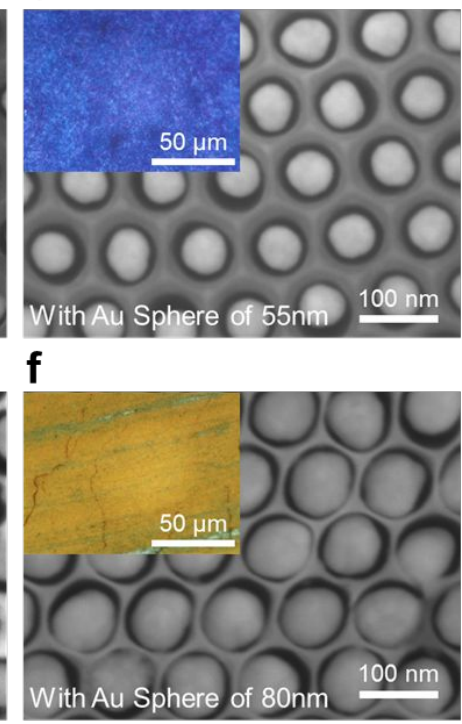

Au sphere size $(\mathrm{nm})$

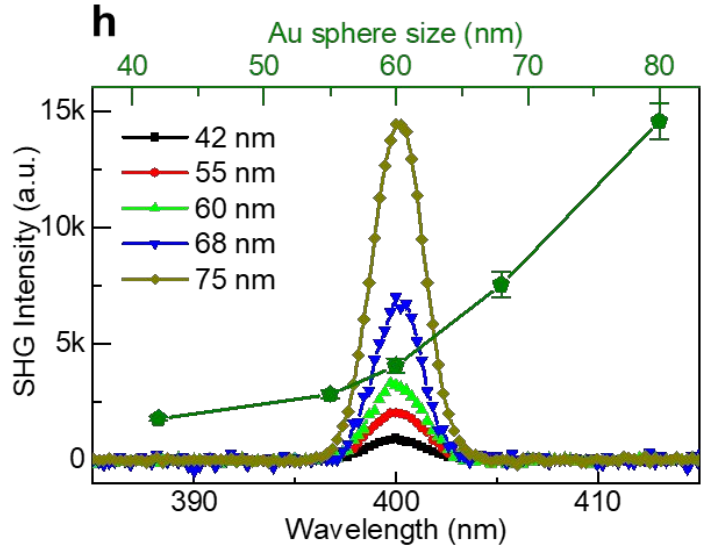

Figure S1. Plasmonic nanostructure array with different size. (a-f) SEM images of the structures with increasing pore/sphere sizes. Optical pictures of each structure are shown in the inset. (g) Extinction spectra of the assembled structures. (h) Corresponding SHG spectra of hybrid structures shown above excited using an $880 \mathrm{~nm}$ pulsed laser. The SHG signal is maximized when the excitation laser wavelength and the SHG emission wavelength match with the double-resonant plasmonic modes supported by the hybrid structures. The plot shows the variation of SHG peak intensity at 440nm with respect to the plasmonic resonances (below) of different hybrid structures (above). 


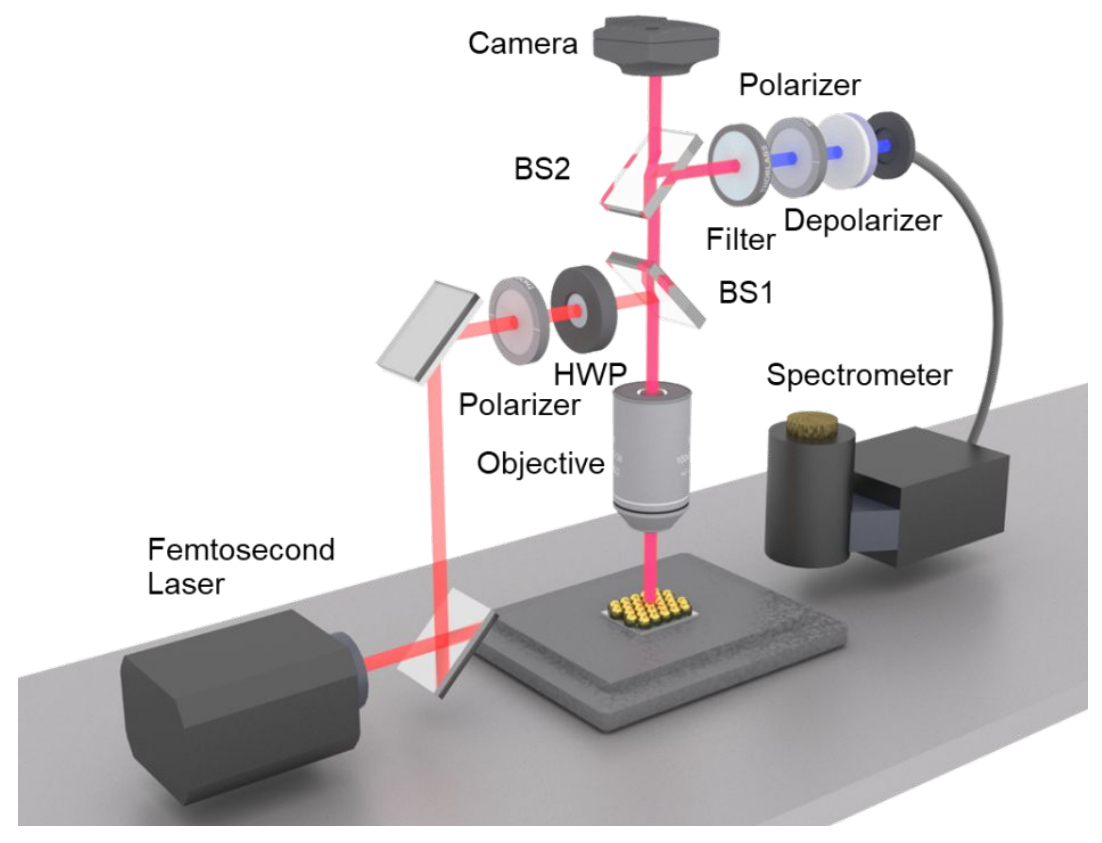

Figure S2. Schematic diagram of the SHG measurement. The optical setup used in the SHG measurement are depicted in Fig. S1. A pulse laser with 140-fs pulse width and $80 \mathrm{MHz}$ repetition rate laser was used to excite the example. A $100 \times$ objective (N.A. $=0.9$, Olympus) was used to focus the fundamental beam and collect the excited SHG signal. The combination of polarizer and HWP (half-wave plate) before BS1 (beam-splitter) is used to change the linear polarization direction of the fundamental beam. The incident beam is blocked by a $694 \mathrm{~nm}$ short-pass filter (semrock), which is set just after the BS2. The polarizer after the short-pass filter functions as a polarization analyzer, while the depolarizer is used to eliminate the signal measurement error caused by the polarization response of the spectrometer. 


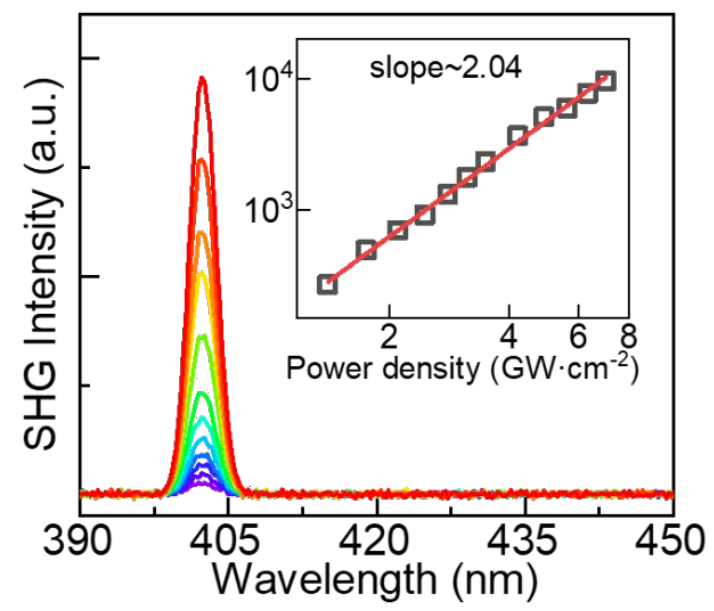

Figure S3. Power dependence of SHG intensity. SHG spectra under different excitation power. Inset: The excitation power dependence of SHG peak intensity in logarithmic coordinates with power density ranging from 1.4 to $7 \mathrm{GW} \cdot \mathrm{cm}^{-2}$, which can be well fitted by a power-law function with coefficient of 2.04 (red curve), confirms the contribution of SHG signals. 

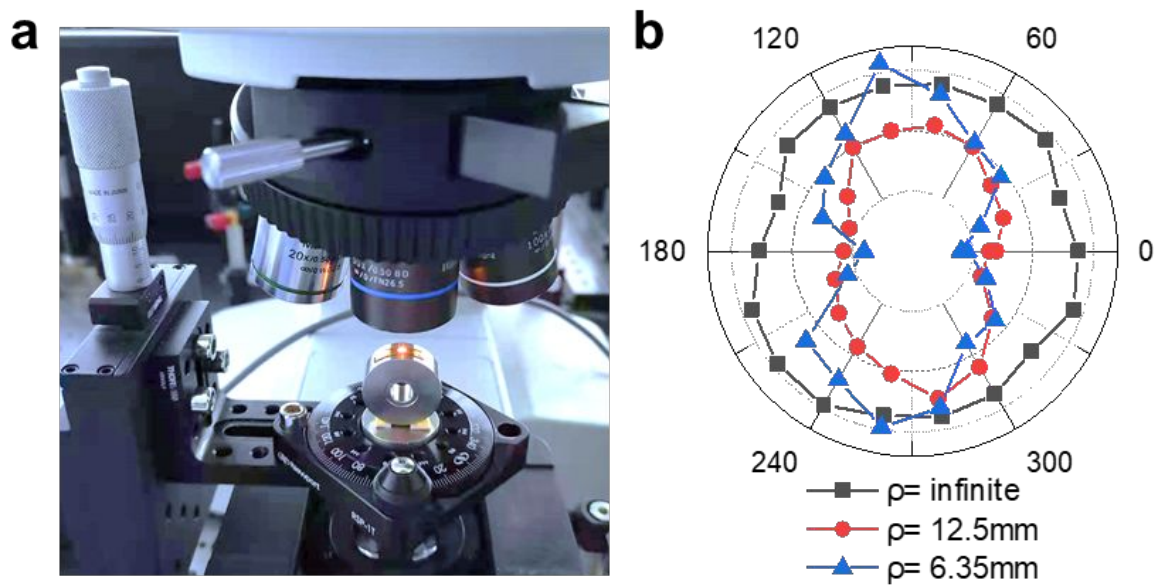

Figure S4 Nonlinear generation from the plasmonic array under applied strain. (a) Photo of optical setup for characterization of nonlinear generation from the plasmonic nanostructure array under mechanical deformation. (b) Polarization-dependent SHG signal for samples under different mechanical deformation with normally incident polarization angles $(\varphi)$ ranging from 0 to $360^{\circ}$. 


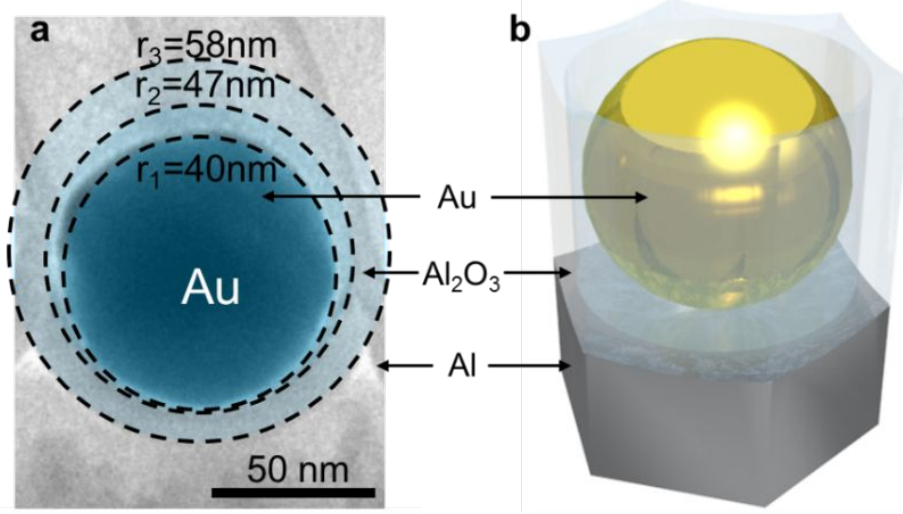

c

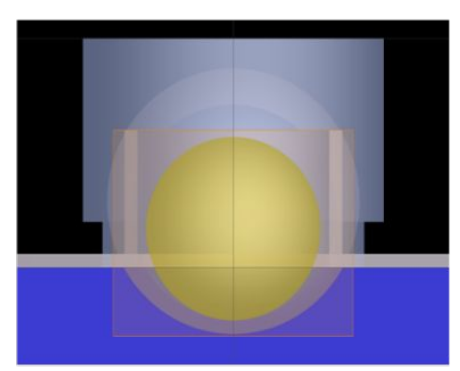

Figure S5. Cross-section TEM pictures of the hybrid structure sample. (a) An $80 \mathrm{~nm}$ size gold nanoparticle was embedded in an $\mathrm{Al}$ convex pore, with a $5 \mathrm{~nm}$ thickness layer of $\mathrm{Al}_{2} \mathrm{O}_{3}$ in the interface. Scale bars: $50 \mathrm{~nm}$. (b) The structure is modeled according to the TEM data in a, (c) the numerical geometry of ball-in-bowl nanostructure. The yellow sphere, gray thin layer and blue substrate represent gold particle, $\mathrm{Al}_{2} \mathrm{O}_{3}$ layer and $\mathrm{Al}$ substrate. The light blue area outside the structure unit is the etch material component, which is used to remove redundant parts and produce a curved surface.

\section{Preparation of the cross-sectional sample for TEM}

The cross-sectional sample for Transmission electron microscopy (TEM) was prepared as follows: Firstly, the sample was embedded with Epon 812 epoxy resin and then solidificated at $60{ }^{\circ} \mathrm{C}$. Secondly, ultrathin sectioning of the sample was performed with an ultramicrotome (Leica EM UC-7) at room temperature. Finally, the ultrathin sectioned sample was transferred onto a TEM grid and allowed for drying at room temperature for the TEM measurement. TEM images were acquired using a FEI Tecnai G2 20 ST microscopes operated at $200 \mathrm{kV}$. 

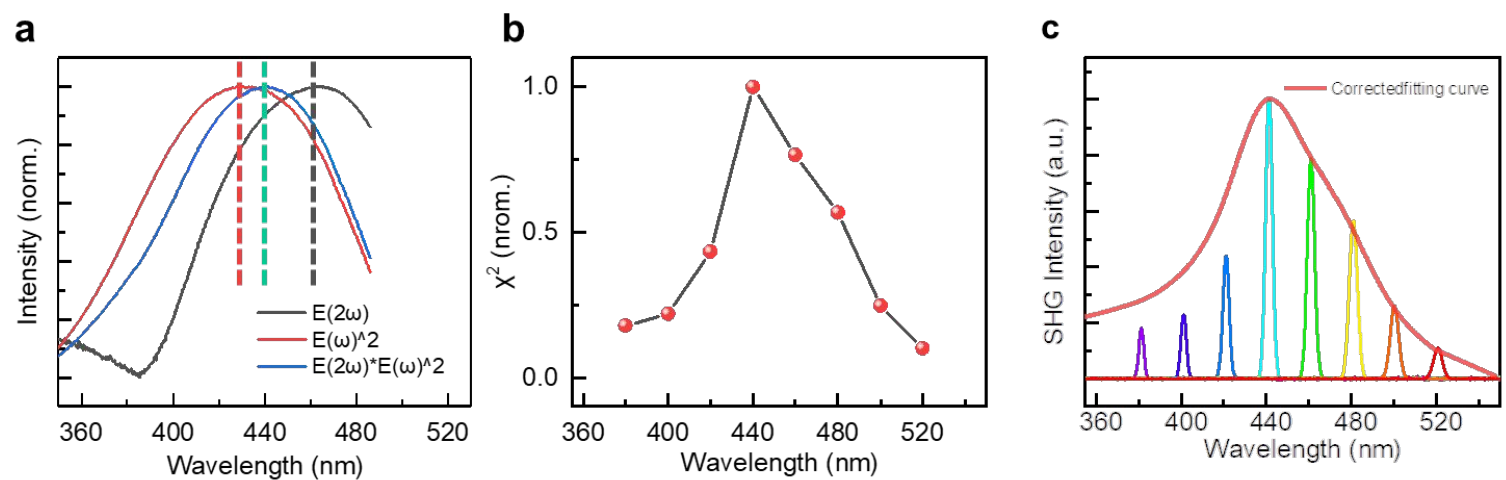

Figure S6. Wavelength dependent SHG from Hybrid structures. (a) Wavelength-resolved enhancement factors of electrical field. Extracted from the extinction spectra, normalized enhancement factor of $E_{n}(\omega), E_{n}(2 \omega)$ and the calculated $E_{n}^{2}(\omega) \cdot E_{n}(2 \omega)$ according to the Lorentz reciprocity theorem are plotted in same wavelength region for an intuitive comparison $^{1-3}$. (b) Dispersion of effective second-order nonlinear susceptibility calculated from the wavelength-dependent SHG spectra. (c) The calculated curve of $E_{n}^{2}(\omega) \cdot E_{n}(2 \omega)$ fits well with the wavelength-dependent SHG distribution, with a maximum $\sim 440 \mathrm{~nm}$. 

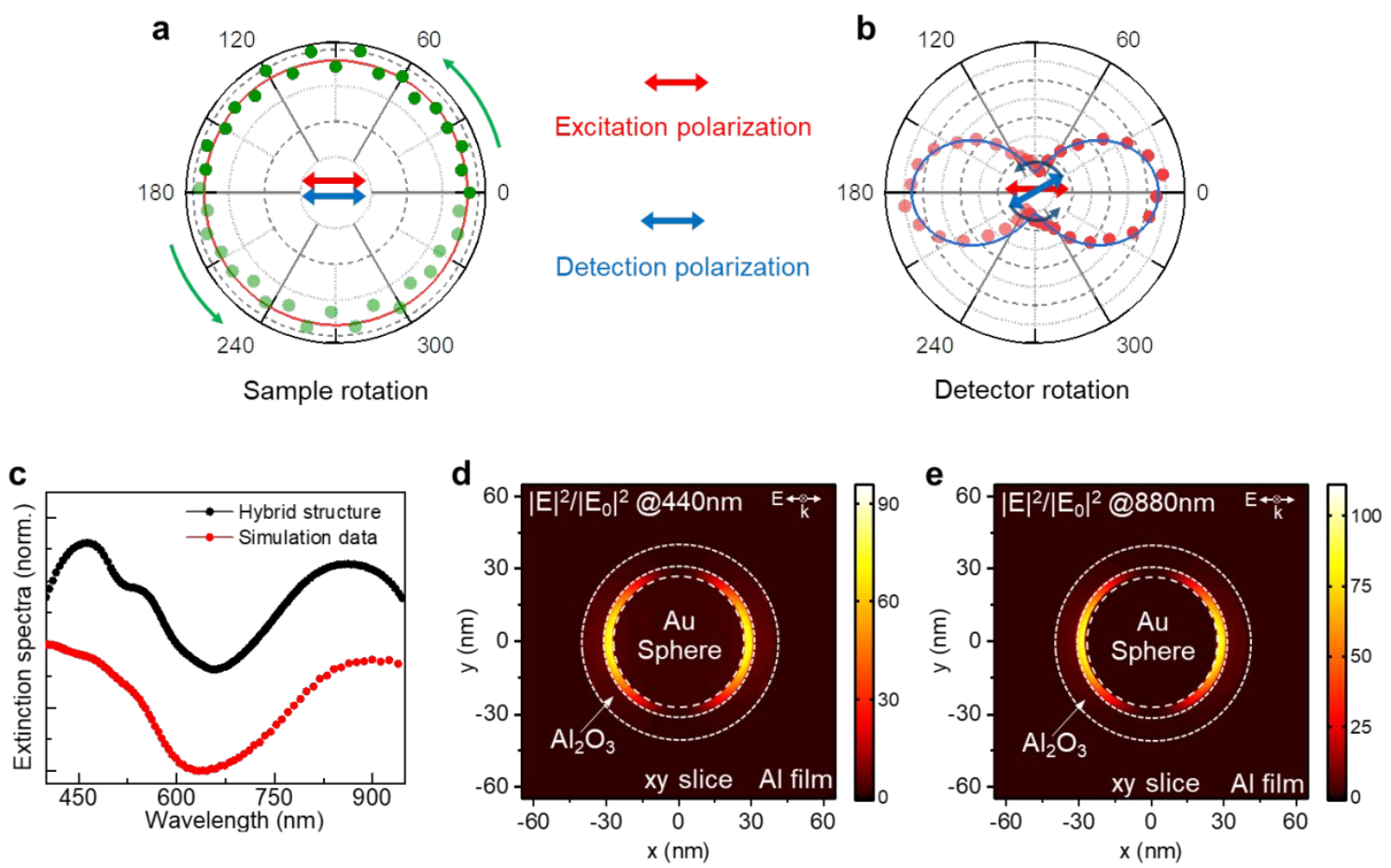

Figure S7. Polarization-dependent characterization of the hybrid nanostructure array. (a-b) Normalized SHG signal intensity at incident wavelength $\lambda=800 \mathrm{~nm}$. In Figure S5a, the sample was rotated from 0 to $180^{\circ}$ to study the anisotropy of structure, with the polarization of excitation laser (red arrow) and detection (blue arrow) unchanged. In Figure S3b, the polarization of detection was rotated from 0 to $180^{\circ}$ to study the anisotropy of radiation polarization, with the polarization of excitation laser and sample unrotated. (c) The simulated extinciton at normal incidence clearly displays the double resonances of hybrid structure. (d-e) Electric near-field in the interlayer of the hybrid structure unit, at the wavelength of SPR mode $(440 \mathrm{~nm})$ and gap mode $(880 \mathrm{~nm})$. 


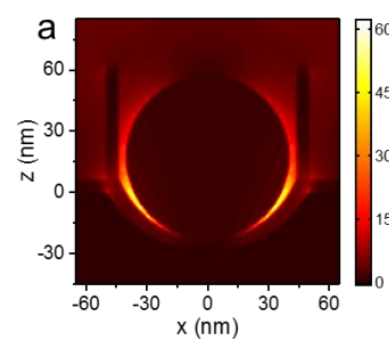

E-field of $\mathrm{Au}-\mathrm{Al}_{2} \mathrm{O}_{3}-\mathrm{Al}$

(Bowl-ball) structure@440

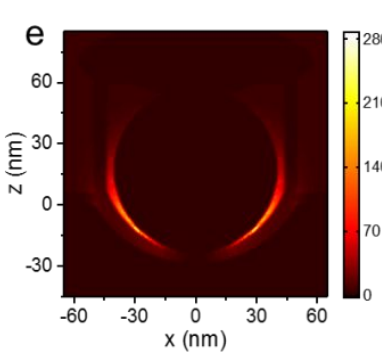

E-field of $\mathrm{Au}-\mathrm{Al}_{2} \mathrm{O}_{3}-\mathrm{Al}$ (Bowl-ball) structure@880

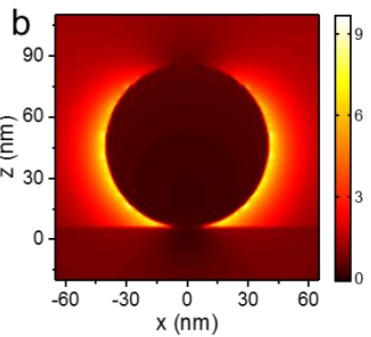

E-field of $\mathrm{Au}-\mathrm{Al}_{2} \mathrm{O}_{3}$ combination@440

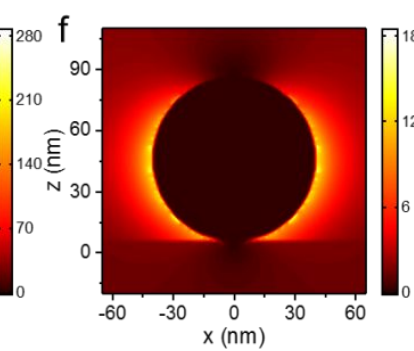

E-field of $\mathrm{Au}-\mathrm{Al}_{2} \mathrm{O}_{3}$ combination@880

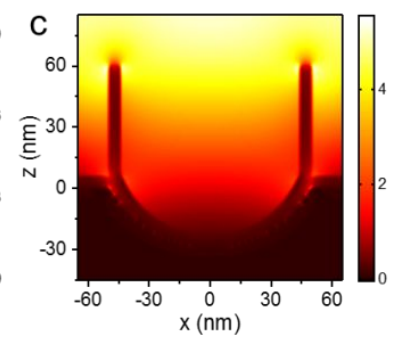

E-field of $\mathrm{Al}_{2} \mathrm{O}_{3}-\mathrm{Al}$ combination@440

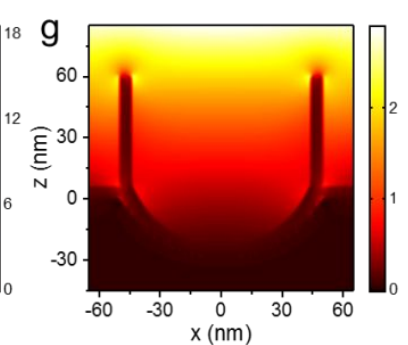

E-field of $\mathrm{Al}_{2} \mathrm{O}_{3}-\mathrm{Al}$ combination@880

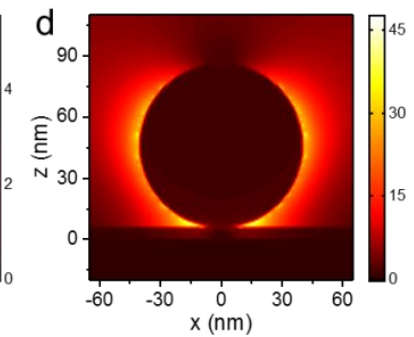

E-field of $\mathrm{Au}-\mathrm{Al}_{2} \mathrm{O}_{3}-\mathrm{Al}$ (NSoM) structure@440

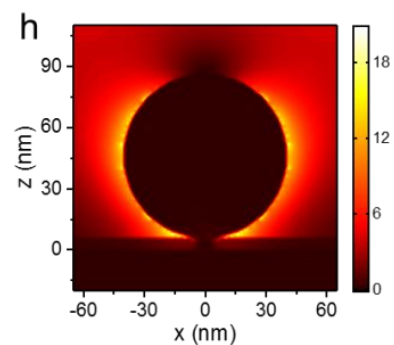

E-field of $\mathrm{Au}-\mathrm{Al}_{2} \mathrm{O}_{3}-\mathrm{Al}$ (NSoM) structure@880

Figure S8. Comparisons in simulated electric field intensity distribution among different hybrid nanostructure. (a-d) The electric field intensity distribution $|E|^{2}$ at second harmonic region of $\mathrm{Au}-\mathrm{Al}_{2} \mathrm{O}_{3}-\mathrm{Al}$ (ball-in-bowl) structure, $\mathrm{Au}-\mathrm{Al}_{2} \mathrm{O}_{3}$ combination, $\mathrm{Al}_{2} \mathrm{O}_{3}-\mathrm{Al}$ combination and $\mathrm{Au}-\mathrm{Al}_{2} \mathrm{O}_{3}-\mathrm{Al}(\mathrm{NPoM})$ structure, respectively. (e-h) The corresponding distribution of different combinations at the fundamental region. 

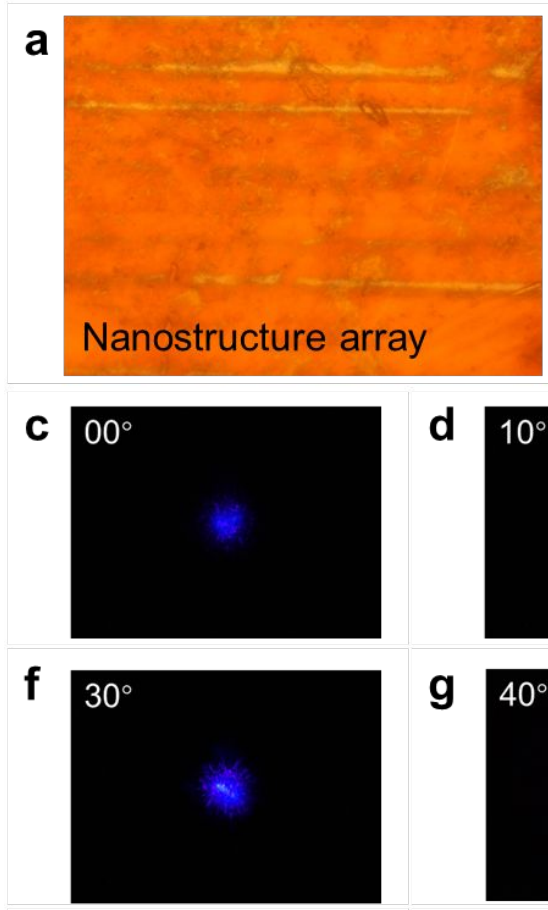

i

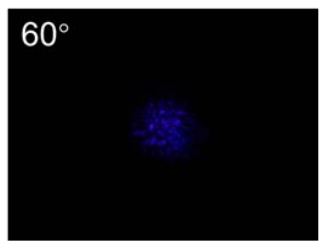

d $10^{\circ}$

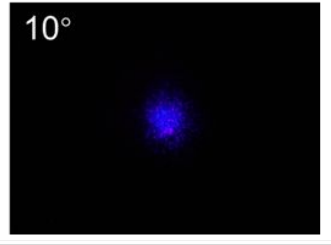

g $40^{\circ}$
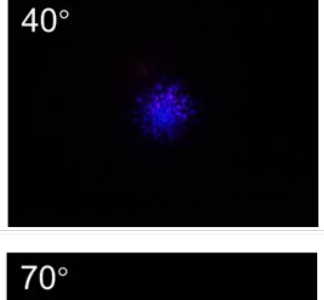

b

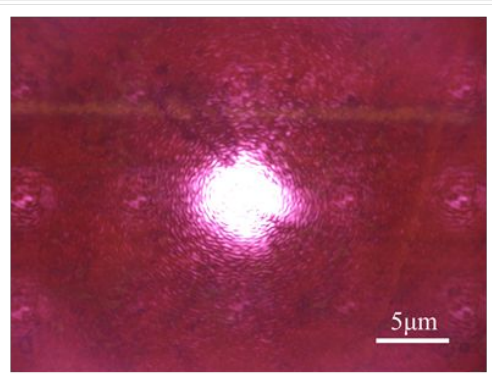

e $20^{\circ}$

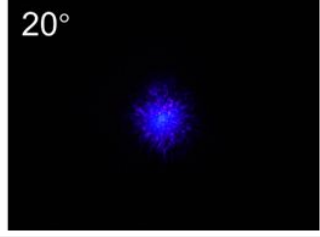

h $50^{\circ}$

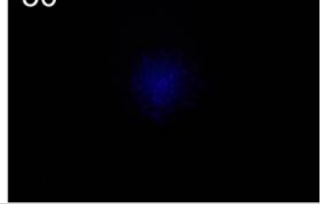

k $80^{\circ}$

Figure S9. Real-space images of SHG from hybrid nanostructure array at different tilt-angle. (a-b) The optical photo of hybrid nanostructure array with the focal spot of the fundamental laser beam. (c-k) With the fundamental excitation beam at $800 \mathrm{~nm}$ blocked by a short-pass filter, real space images of SHG signal from hybrid nanostructure array at different tilt-angles were recorded in CMOS camera. 
a

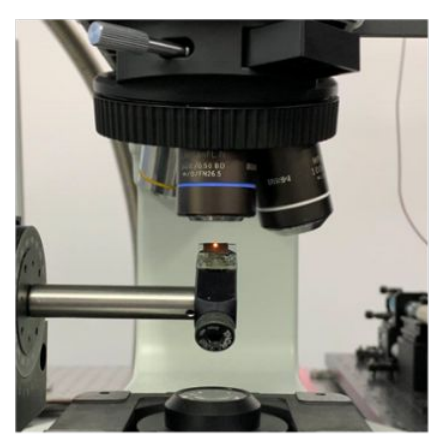

b

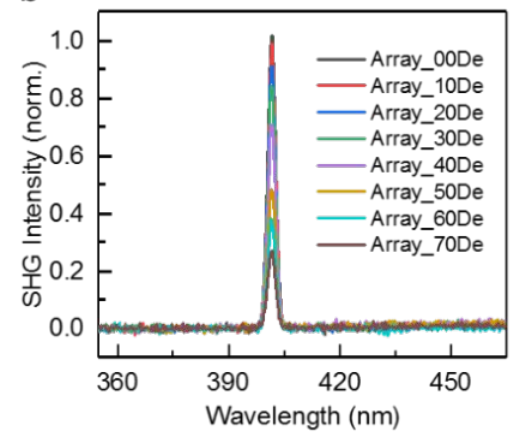

C

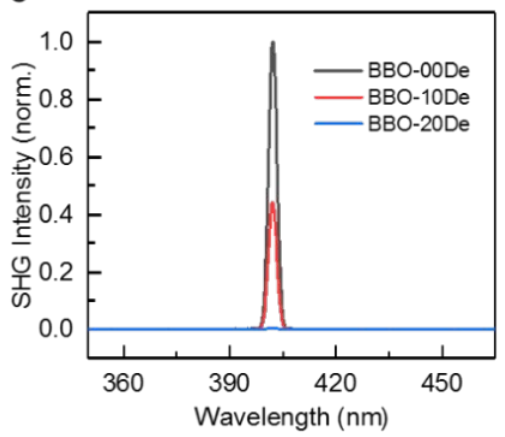

Figure S10. Tilt-dependent SHG signals of different samples. (a) Photo of optical setup for characterization of tilt-angle dependent SHG. (b-c) Second harmonic signal generated from the hybrid nanostructure array and commercial BBO crystal (CASTECH) at $\lambda=400 \mathrm{~nm}$ for tilt-angles ranging from 0 to $70^{\circ}$. 


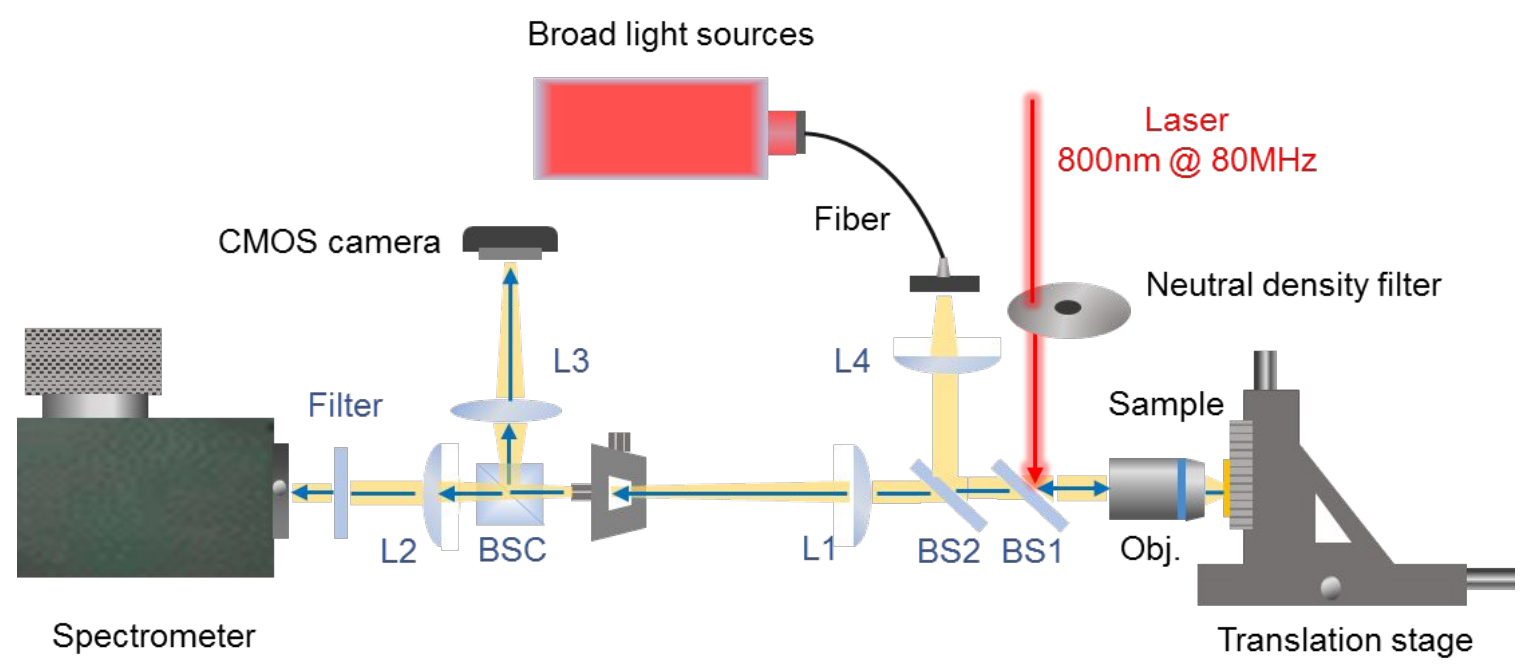

Figure S11. Optical setup for angle-resolved spectroscopy by spatially-resolved Fourier imaging. Pulse laser and white light are introduced by the beam-splitters BS1 and BS2. Obj. is a $50 \times$ objective lens (Olympus MPLFLN $50 \times$ N.A. $=0.8$ ). L1, L2 and L3 are lenses for transformation between real-space image and fourier image, while L4 is a lens for collimation of white light. In order to observe the fourier image and corresponding real-space image simultaneously, a BSC (beam splitter cube) is set in the light path. 

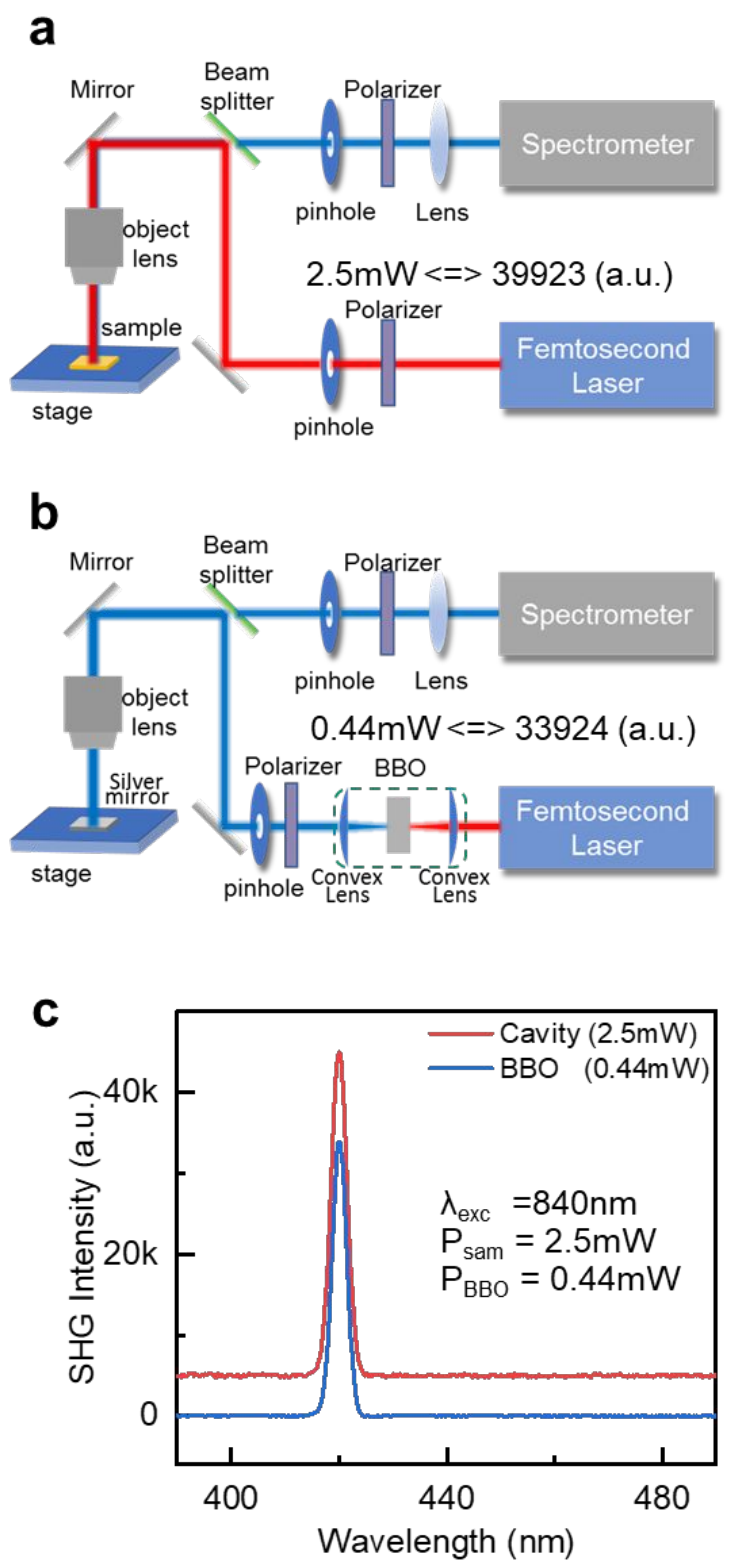

Figure S12. Effective second-order nonlinear susceptibility $\chi^{(2)}$ of the structure array. (a-b) Schematic diagram of the optical paths for calculating the effective second-order nonlinear susceptibility with the commercial BBO crystal (CASTECH) used as reference. (c) The comparison of SHG intensity from samples excited at the same wavelength was conducted when using the commercial BBO crystal as the reference. 
Supplementary Note 1. Nonlinear response of the hybrid nanostructure array.

The effective second-order nonlinear susceptibility tensor of the device $\left(\chi^{(2) \text { eff }}\right)$ may be related to these field distributions by using the Lorentz reciprocity theorem ${ }^{4}$, yielding the expression for the general ijk element

$$
\chi_{i j k}^{(2) e f f}=\chi\left(\sum_{z z}^{(2)} \frac{\int_{V} E_{z(i)}^{2 \omega}(x, y, z) E_{z(j)}^{\omega}(x, y, z) E_{z(k)}^{\omega}(x, y, z) d V}{E_{i(i n c)}^{2 \omega} E_{j(i n c)}^{\omega} E_{k(i n c)}^{\omega} V}\right.
$$

where $E_{z(i)}^{\omega}$ and $E_{z(i)}^{2 \omega}$ are the local $E_{z}$ field components in the structure array induced by i-polarized incident waves $E_{i(\text { inc })}^{\omega}$ and $E_{i(\text { inc })}^{2 \omega}$, respectively, at fundamental and second harmonic frequencies, respectively, $\mathrm{i}$ can be $\mathrm{x}, \mathrm{y}$ or $\mathrm{z}$, and the integration covers the volume (V) of the hybrid nanostructure in the unit cell.

Because of the centrosymmetry-breaking limit according to electric-dipole approximations for isotropic materials (gold), different elements of the susceptibility tensor for our nanostructure unit can be experimentally treated as a single nonvanishing element (surface normal component $\left.\chi^{\left.(2)_{n}\right)}\right)^{5}$, the expression (1) can be simplified as

$$
I_{S H G} \propto|L(2 \omega)|^{2}|L(\omega)|^{4}
$$

where $\mathrm{L}(\omega)$ refers to the local field enhancement through $L(\omega)=E_{\mathrm{loc}}(\omega) / \mathrm{E}(\omega)$, in which $E_{\mathrm{loc}}(\omega)$ and $E(\omega)$ are the local field amplitude and the electric far-field amplitude at fundamental and second harmonic frequencies, respectively. The equation (2) demonstrates that efficient SHG intensities vary super-linearly with associated local-field distributions at both frequencies. By characterizing the extinction spectrum of the structure, the corresponding distribution of local field amplitude was obtained and the simulated SHG conversion efficiency distribution was then calculated according to the equation (2) (see Figure S2a). The wavelength-dependent SHG intensity obtained by the experiment and the calculation results of the equation are plotted in Figure S2b. in which the calculation results fit well with the experimental data and the strongest SHG conversion is obtained at 440nm (excitation at $880 \mathrm{~nm}$ ) in both plots. This indicates that SHG conversion in our device derives from the double resonance modes at both frequencies realized by the combination of several components. 
Supplementary Note 2. Analysis of effective second-order nonlinear susceptibility.

The effective second-order nonlinear susceptibility of hybrid structure was calculated based on the measurement of SHG performed on hybrid structure with a commercial BBO crystal as a reference sample. Both the hybrid structure and BBO crystal were tested in the same setup to avoid the influence of instrument difference. Figure S7a, $\mathbf{b}$ displayed the schematic diagram of the optical paths for SHG measurements. For an $\mathrm{o}+\mathrm{o} \rightarrow \mathrm{e}$ SHG process, the light conversion relation can be described as follow ${ }^{6}$ :

$$
\mathrm{P}_{2 \omega}=\frac{2 \pi^{2}\left(\chi_{\mathrm{eff}}^{(2)}\right)^{2} \mathrm{~L}^{2} \mathrm{P}_{\omega}^{2}}{\varepsilon_{0} \mathrm{cn}_{\omega}^{2} \mathrm{n}_{2 \omega} \lambda_{2 \omega}^{2} \mathrm{~A}} \cdot \frac{\sin ^{2}\left(\Delta \mathrm{k}_{2}^{\mathrm{L}}\right)}{\Delta \mathrm{k}_{2}^{\mathrm{L}}}
$$

Here in equation (3), $\mathrm{P}_{2 \omega}$ and $\mathrm{P}_{\omega}$ represent the second harmonic emission power and the excitation power for the nonlinear process, $\chi_{\text {eff }}$ is the effective second-order nonlinear susceptibility, $L$ is the length of the sample, $\varepsilon_{0}$ is the vacuum dielectric constant, $\mathrm{c}$ is the speed of light in vacuum and $\mathrm{A}$ is the laser beam illuminance area. Neglecting the difference in the refractive index $\left(n_{\omega}\right.$ and $\left.n_{2 \omega}\right)$ and the phase-matching condition of hybrid nanostructure, the equation (3) can be simplified for measurement of the effective second-order susceptibility under same experimental conditions as

$$
\frac{\chi_{e f f(\operatorname{Sam} 1)}^{(2)}}{\chi_{e f f(\operatorname{Sam} 0)}^{(2)}}=\frac{L_{\text {Sam } 0}}{L_{\text {Sam } 1}} \cdot \frac{P_{\text {Sam } 0, \omega}}{P_{\text {Sam } 1, \omega}} \cdot \sqrt{\frac{A_{\text {Sam } 1} P_{\text {Sam } 1,2 \omega}}{A_{\text {Sam } 0} P_{\text {Sam } 0,2 \omega}}}
$$

The Length of hybrid structure can be directly obtained from the cross-section TEM measurement. The picture in Figure 1d shows that the total vertical height of hybrid structure is about $120 \mathrm{~nm}$, which can be taken as the effective interference length since it is much shorter than the wavelength of the incident laser. The effective second-order susceptibility of Commercial $\mathrm{BBO}$ bulk crystal is set as $2.04 \mathrm{pm} / \mathrm{V}^{7}$. Considering that the total conversion efficiency of the thick BBO crystal is too strong, different incident laser power values were used to excite the hybrid nanostructure array and the BBO crystal, respectively, in order to get the SHG intensity of the same magnitude (see Figure S7c). With the experimental data concluded above corrected by the transmittance of optical components (about 90\%) used in measurements, we can roughly estimate the effective second-order susceptibility of the hybrid structure to be $140 \mathrm{pm} / \mathrm{V}$. 


$$
\begin{aligned}
& \chi_{\mathrm{eff}(\mathrm{Sam})}^{(2)}=\frac{2.5 \mathrm{~mm}}{120 \mathrm{~nm}} \cdot \frac{0.44 \mathrm{~mW} \times 0.9}{2.5 \mathrm{~mW}} \cdot \sqrt{\frac{(3 \mu \mathrm{m})^{2} \times(0.9)^{-5}}{(500 \mu \mathrm{m})^{2} \times(0.9)^{-5} \times\left(\frac{1}{20}\right) \times(0.8)^{-3}}} \cdot \chi_{\mathrm{eff}(\mathrm{BBO})}^{(2)} \\
& =68.8 \times \chi_{e f f(B B O)}^{(2)}=140 \mathrm{pm} / \mathrm{V}
\end{aligned}
$$

SHG conversion efficiency is another important parameter to measure the nonlinear performance of our nanostructure array, which is conducted in the equation (5).

$$
\eta=\frac{P_{S H G}}{P_{E x c}}=8 \pi^{2} L^{2}\left(\chi^{(2)}\right)^{2} P_{E x c} / n_{\omega}^{2} n_{2 \omega} \lambda_{\omega}^{2} A c \varepsilon_{0}
$$

In equation above, $P_{E x c}$ and $P_{S H G}$ are excitation power and $\mathrm{SH}$ power, respectively. Based on the parameters used in previous calculation, the SHG conversion efficiency was then achieved as $\eta_{\mathrm{SHG}}=3.84 \times 10^{-9}$ under $7 \mathrm{GW} \mathrm{cm}^{-2}$.

$$
\begin{aligned}
& \eta=\frac{8 \pi^{2} \times(120 \mathrm{~nm})^{2} \times(140 \mathrm{pm} / \mathrm{V})^{2} \times(2.5 \mathrm{~mW})}{1^{2} \times 1 \times(880 \mathrm{~nm})^{2} \times\left(\pi \times\left(\frac{3 \mu \mathrm{m}}{2}\right)^{2}\right) \times\left(3 \times 10^{8} \mathrm{~m} / \mathrm{s}\right) \times\left(8.854 \times 10^{-12} \mathrm{~F} / \mathrm{m}\right)} \\
& =3.84 \times 10^{-9} .
\end{aligned}
$$




\section{REFERENCES}

(1) Roke, S.; Bonn, M.; Petukhov, A. V., Nonlinear Optical Scattering: The Concept of Effective Susceptibility. Phys. Rev. B 2004, 70 (11).

(2) O'Brien, K.; Suchowski, H.; Rho, J.; Salandrino, A.; Kante, B.; Yin, X.; Zhang, X., Predicting Nonlinear Properties of Metamaterials from the Linear Response. Nat. Mater. 2015, 14 (4), 379-83.

(3) Wang, F.; Martinson, A. B. F.; Harutyunyan, H., Efficient Nonlinear Metasurface Based on Nonplanar Plasmonic Nanocavities. ACS Photonics 2017, 4 (5), 1188-1194.

(4) Balanis, C. A., Advanced Engineering Electromagnetics; Wiley: New York, 1989.

(5) Shen, S.; Meng, L.; Zhang, Y.; Han, J.; Ma, Z.; Hu, S.; He, Y.; Li, J.; Ren, B.; Shih, T. M.; Wang, Z.; Yang, Z.; Tian, Z., Plasmon-Enhanced Second-Harmonic Generation Nanorulers with Ultrahigh Sensitivities. Nano Lett. 2015, 15 (10), 6716-21.

(6) Shen, Y. R., The Principles of Nonlinear Optics; Wiley-Interscience, NY, 1984.

(7) Alford, W. J.; Smith, A. V., Wavelength Variation of the Second-Order Nonlinear Coefficients of $\mathrm{KNbO}_{3}, \mathrm{KTiOPO}_{4}, \mathrm{KTiOAsO}_{4}, \mathrm{LiNbO}_{3}, \mathrm{LiIO}_{3}, \beta-\mathrm{BaB}_{2} \mathrm{O}_{4}, \mathrm{KH}_{2} \mathrm{PO}_{4}$, and $\mathrm{LiB}_{3} \mathrm{O}_{5}$ Crystals: a Test of Miller Wavelength Scaling. J. Opt. Soc. Am. B 2001, 18 (4), 524-533. 